\title{
Compact Feynman rules for Majorana fermions
}

\author{
A. DENNER \\ CERN, Geneva, Switzerland \\ H. ECK, O. HAHN, J. KÜBLBECK \\ Physikalisches Institut, University of Würzburg, Germany
}

\begin{abstract}
:
We present simple algorithmic Feynman rules for Majorana fermions. Insisting on a fermion flow through the graphs along fermion lines we only need the familiar Dirac propagator and only vertices without explicit charge-conjugation matrices; moreover, we get the correct relative signs between different interfering Feynman graphs as in the case of Dirac fermions.
\end{abstract}

Supersymmetric gauge theories involve Majorana fermions. The fact that Majorana fields are self-conjugate implies that there exist Wick contractions of these fields which are different from those of Dirac fields. Together with the anticommutativity of fermion fields this obscures the determination of the relative sign of interfering Feynman graphs.

Feynman rules for Majorana fermions were given in Refs. [1, 2, 3, 4]. However, those Feynman rules seem to be either unnecessarily complicated or do not allow the determination of the relative sign of interfering Feynman graphs directly from the diagrams.

We derive Feynman rules for Majorana (and Dirac) fermions which are close to the rules for Dirac fermions. Since the fermion number flow is violated we introduce a continuous fermion flow, i.e. an (arbitrary) orientation of each fermion line. Based on this fermion flow we can form chains of Dirac matrices and determine the relative sign of interfering Feynman graphs as one does for Dirac fermions. We only need the well-known Dirac propagator for all fermions, and we have to introduce in general two analytical expressions for each vertex (one for the two possible orientations), which is still less than in the usual treatment.

To be more specific we consider a typical coupling term $\mathcal{L}_{I}=\bar{\chi} \Gamma \chi$ where each $\chi$ can be a Dirac or a Majorana fermion and $\Gamma$ denotes a generic fermionic interaction including Dirac matrices, coupling constants $h_{a b c}^{i}$ and boson fields:

$$
\bar{\chi} \Gamma \chi=h_{a b c}^{i} \bar{\chi}_{a} \Gamma_{i} \chi_{b} \Phi_{c} .
$$

The field $\Phi$ summarizes scalar and vector fields and $\Gamma_{i}=1, i \gamma_{5}, \gamma_{\mu} \gamma_{5}, \gamma_{\mu}, \sigma_{\mu \nu}$.

To be able to follow the introduced fermion flow along the oriented fermion line it may be necessary to rewrite $\mathcal{L}_{I}$ in the equivalent "reversed" form

$$
\mathcal{L}_{I}=\overline{\widetilde{\chi}} \Gamma^{\prime} \tilde{\chi}
$$


with the charge conjugate fields

$$
\tilde{\chi}=C \bar{\chi}^{T}, \bar{\chi}=-\chi^{T} C^{\dagger}
$$

and

$$
\Gamma^{\prime}=C \Gamma^{T} C^{\dagger}
$$

Using the properties of the charge conjugation matrix $C$

$$
C^{\dagger}=C^{-1}, \quad C^{T}=-C, \quad C \Gamma_{i}^{T} C^{-1}=\eta_{i} \Gamma_{i} \quad \text { (no summation over } i \text { ) }
$$

with

$$
\eta_{i}=\left\{\begin{aligned}
1 \text { for } \Gamma_{i} & =1, i \gamma_{5}, \gamma_{\mu} \gamma_{5} \\
-1 & \text { for } \Gamma_{i}=\gamma_{\mu}, \sigma_{\mu \nu}
\end{aligned}\right.
$$

we obtain

$$
\overline{\widetilde{\chi}} \Gamma^{\prime} \tilde{\chi}=h_{a b c}^{i} \eta_{i} \overline{\widetilde{\chi}}_{b} \Gamma_{i} \tilde{\chi}_{a} \Phi_{c}
$$

If both $\chi$ are Majorana fermions (7) implies $\eta_{i} h_{a b c}^{i}=h_{b a c}^{i}$ for all $i$, i.e. $\Gamma=\Gamma^{\prime}$.

For the spinors we have

$$
v(p, s)=C \bar{u}^{T}(p, s) \quad u(p, s)=C \bar{v}^{T}(p, s)
$$

where $p$ and $s$ denote momentum and polarization, respectively, and for the propagator $S(p)$

$$
C S(p) C^{-1}=S(-p) \text {. }
$$

A detailed derivation including a more complete set of examples and a comparison with Refs. [1, 2, 3, 4] will be given in [5], where we also show that our Feynman rules naturally apply to theories containing explicit charge-conjugate fermion fields.

So we can write down our Feynman rules:

Fermions are denoted by solid lines. For Dirac fermions, each line carries an arrow which indicates the fermion number flow. Majorana lines do not carry arrows.

The fermionic vertices are read off from the Lagrangian as usual, but for every vertex containing fermions we need two expressions, the direct one $(\Gamma)$ and the "reversed" one $\left(\Gamma^{\prime}\right)$. For a pure Majorana fermion vertex there is only one expression because $\Gamma=\Gamma^{\prime}$.

With respect to the fermion number flow of Dirac fermions there exists the usual propagator $S(p)$ and the "reversed" one $S^{\prime}(p)=S(-p)$ as well the usual spinors and their "reversed" counterparts. For Majorana fermions there are only the usual propagator and spinors and no reversed ones (see Figs. 2 and 3). With respect to the fermion flow the "reversed" spinors and propagators equal the usual ones and there is only one propagator and the usual spinors ${ }^{1}$.

With this rules the Feynman amplitudes are obtained as follows:

- Draw all possible Feynman diagrams for a given process.

\footnotetext{
${ }^{1}$ The usual Feynman rules for propagators and external fermions depend on the relative orientation of fermion number flow and momentum. Our Feynman rules are obtained from the usual ones when substituting the fermion number flow by the fermion flow using the same set of analytical expressions.
} 


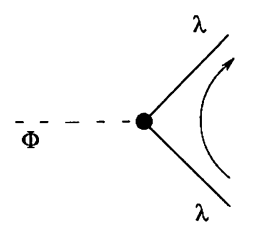

$i \Gamma$
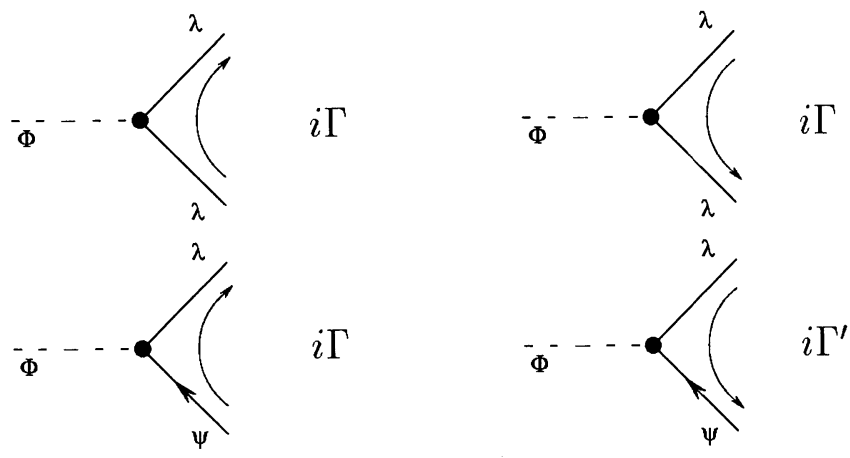

$i \Gamma$
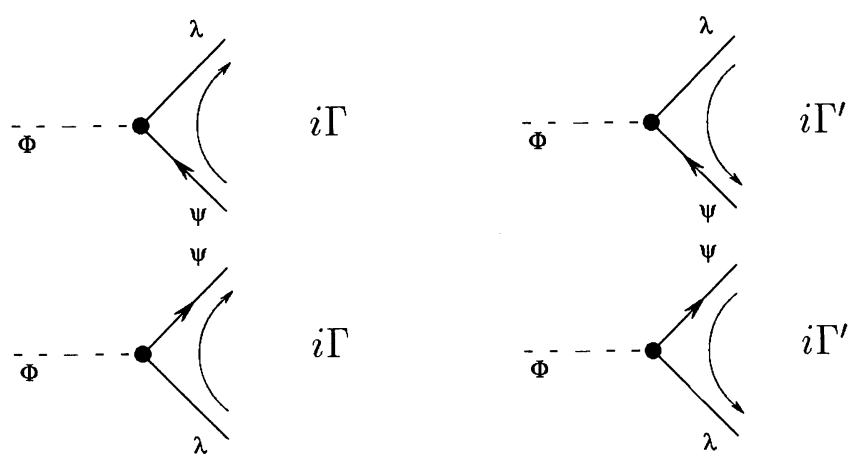

$i \Gamma$
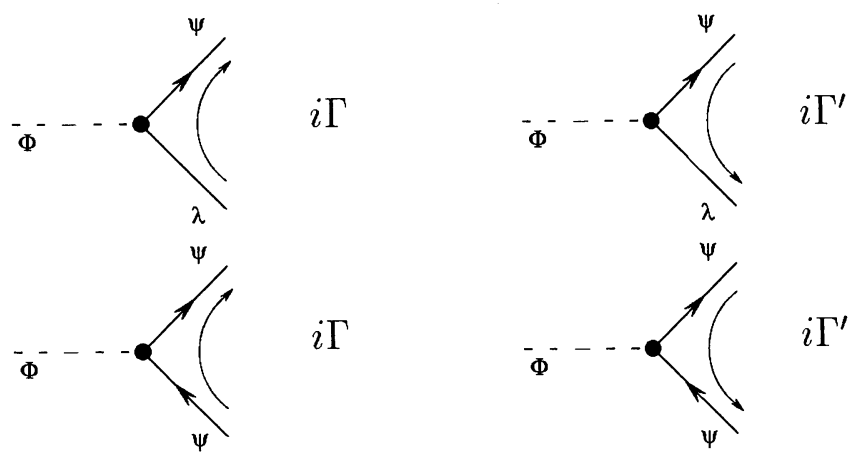

$i \Gamma^{\prime}$

Figure 1: The Feynman rules for fermionic vertices with orientation (thin arrows)

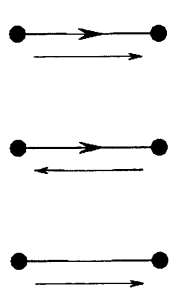

$$
\begin{aligned}
& i S(p) \\
& i S(-p) \\
& i S(p)
\end{aligned}
$$

Figure 2: The Feynman rules for fermion propagators with orientation (thin arrows). The momentum $p$ flows from left to right.

- Fix an arbitrary orientation (fermion flow) for each fermion chain.

- Start at an external leg (for closed loops at some arbitrary propagator) and write down the Dirac matrices proceeding opposite to the chosen orientation (fermion flow) through the chain.

- For each internal propagator, external line and vertex insert the appropriate analytic expression as given in Figs. 1, 2 and 3 corresponding to the chosen fermion flow. This implies that if the orientation is opposite to the flow of fermion number the reversed vertices $\Gamma^{\prime}$, propagators $S(-p)$ and spinors have to be taken.

- Multiply by a factor $(-1)$ for every closed loop. 


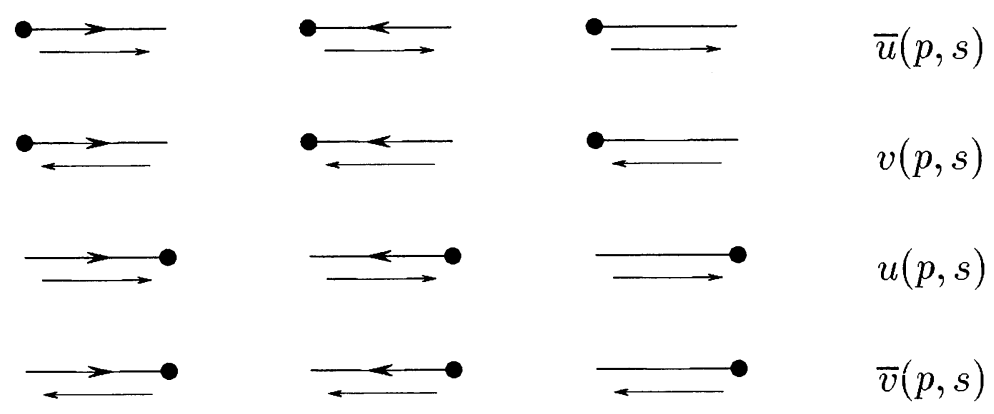

Figure 3: The Feynman rules for external fermion lines with orientation (thin arrows). The momentum $p$ flows from left to right.

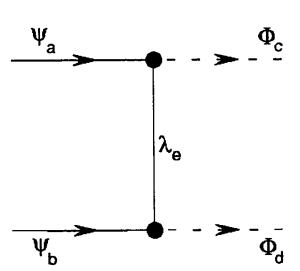

a)

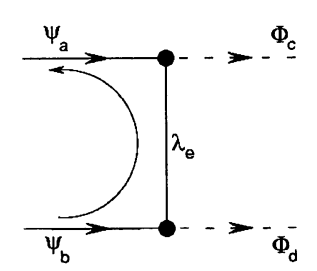

b)

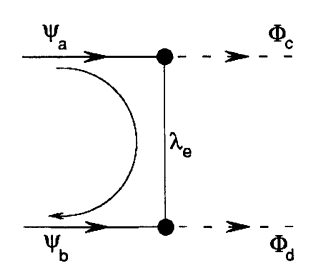

c)

Figure 4: Feynman diagram contributing to $\psi \psi \rightarrow \Phi \Phi$

- Multiply by the permutation parity of the spinors in the obtained analytical expression with respect to some reference order.

- As far as the determination of the combinatorial factor is concerned, Majorana fermions behave exactly like real scalar or vector fields.

Note, that the analytical expressions are independent of the chosen orientation (fermion flow).

We illustrate the application of our Feynman rules by giving one example. We consider the contribution to the process $\psi \psi \rightarrow \Phi \Phi$ via Majorana exchange depicted in Fig. 4 resulting from the interaction Lagrangian $\mathcal{L}_{I}=h_{a b c}^{i} \bar{\lambda}_{a} \Gamma_{i} \psi_{b} \Phi_{c}$ where $\lambda_{a}$ and $\psi_{b}$ denote the Majorana and the Dirac fermions, respectively.

With the help of our Feynman rules we can immediately write down the expression for the amplitude with arbitrary fermion flow. For the orientation in Fig. 4b we get

$$
i \mathcal{M}=-i \bar{v}_{a} \Gamma_{i}^{\prime} S\left(p_{c}-p_{a}\right) \Gamma_{j} u_{b} h_{e a c}^{i} h_{e b d}^{j}
$$

and for the reversed fermion flow (Fig. 4c)

$$
i \mathcal{M}^{\prime}=(-1)(-i) \bar{v}_{b} \Gamma_{j}^{\prime} S\left(p_{a}-p_{c}\right) \Gamma_{i} u_{a} h_{e a c}^{i} h_{e b d}^{j}=i \mathcal{M} .
$$


The sign is determined from the permutation parity of the spinors with respect to a reference order. Fixing the reference order as $(a, b)$ yields the explicit factor $(-1)$ in (10b). The equivalence of (10a) and (10b) can be easily shown using (4), (8) and (9).

The Feynman rules quoted above apply to any order of perturbation theory. Note that a self energy loop with two identical Majorana fermions receives the usual combinatorial factor $\frac{1}{2}$ for identical particles.

To summarize we state that our Feynman rules - based on a well-defined fermion flow - are simple and algorithmic. They resemble closely the usual ones for Dirac fermions and do not involve the charge-conjugation matrix explicitly. All sign ambiguities have disappeared and the relative sign of interfering Feynman diagrams is determined exactly as in the case of Dirac fermions. This set of rules facilitates practical calculations considerably. Furthermore they can easily be implemented into symbolic Feynman diagram generators.

\section{References}

[1] S.K. Jones and C.H. Llewellyn Smith, Nucl. Phys. B217 (1983) 145.

[2] H.E. Haber and G.I. Kane, Phys. Rep. 117 (1985) 76.

[3] E.I. Gates and K.L. Kowalski, Phys. Rev. D37 (1988) 938.

[4] J. Gluza and M. Zrałek, Phys. Rev. D45 (1992) 1693.

[5] A. Denner, H. Eck, O. Hahn and J. Küblbeck, CERN-TH.6549/92. 